\title{
The Self-Coherent Camera : a focal plane sensor for EPICS ?
}

\author{
Pierre Baudoz ${ }^{1,2, a}$, Marion Mas ${ }^{1,2}$, Raphael Galicher ${ }^{1,2}$, and Gerard Rousset ${ }^{1,2}$ \\ 1 LESIA, Observatoire de Paris, CNRS, Université Pierre et Marie Curie Paris 6 and Université Denis \\ Diderot Paris7 - - 5, place Jules Janssen, 92195 Meudon, France. \\ 2 Groupement d'Intérêt Scientifique Partenariat Haute Résolution Angulaire Sol Espace (PHASE) \\ between ONERA, Observatoire de Paris, CNRS and Université Denis Diderot Paris 7
}

\begin{abstract}
In the framework of the European Extremely Large Telescope, EPICS is an ambitious instrument devoted to the direct detection and the characterization of extrasolar planets. Since the performance of such an instrument is limited by wavefront errors, the use of extreme Adaptive Optic (AO) systems is mandatory. Studies for future planet finder instruments such as SPHERE/VLT or GPI/GEMINI show that a strong limitation of the performance of a planet finder is the differential aberrations that are not measured by the wavefront sensor, which is physically separated from the common optics by a beam splitter. We propose here to efficiently estimate these aberrations by directly measuring the wavefront errors in the final science image. To do so, we propose to couple the foreseen extreme AO system at high speed of EPICS with a low speed Self-Coherent Camera (SCC) which can measure directly the differential aberrations at the final science focal plane. The SCC which is based on the principle of light coherence estimates the wavefront errors by spatially encoding the speckles with fringes in the final image. After recalling the principle of the SCC, we present simulation results of the SCC performance in the context of EPICS.
\end{abstract}

\section{Introduction}

Exoplanets are typically $10^{7}$ to $10^{10}$ fainter than their host and are often located within a fraction of an arcsecond from their star. Numerous coronagraphs have been proposed to reduce the overwhelming light of a star to obtain a direct imaging of its planet [1-4]. But performance is limited by wavefront errors in the upstream beam for all these coronagraphs and the final focal plane image is dominated by stellar speckles. The effect of most of these aberrations can be corrected by adaptive optics (AO) or eXtreme AO (XAO [5]) but the uncorrected part generates quasi-static speckles, which limit the image contrast [6]. Differential imaging techniques can be used to estimate and reduce this speckle noise. Several solutions exist based on spectral characteristics [7], polarization states [8], differential rotation in image [9], or incoherence between stellar and companion lights [10]. But as shown in Cavarroc et al. [11], any small difference between the images which are compared in these techniques limits strongly their efficiency. The more appealing solution, before using one of these data processing techniques, is to actively suppress quasi-static wavefront errors so that a first speckle reduction is achieved and differential imaging techniques have less work to do. Because of the low level of aberrations that must be achieved (a few nanometers), the best solution is to measure for phase and amplitude errors directly in the final science image to avoid differential errors introduced by classical wavefront sensor (ShackHartmann for example). Codona \& Angel [12] suggest using the incoherence between companion and stellar lights and use a modified Mach-Zender interferometer to encode the stellar speckles. The instrument we propose, the Self Coherent Camera (SCC), is based on the same property and uses Fizeau interferences. The principle and the capabilities of the Self Coherent Camera have already been described [13-16] but mostly in the context of space-based observations. In this paper, we study the capability of the SCC as a slow wavefront sensor to measure the static aberrations after an extreme AO system on a ground-based telescope. This simulation is made in the framework of the planet finder of the European Extremely Large Telescope (E-ELT), which is called EPICS [17].

\footnotetext{
a e-mail: pierre.baudoz@obspm.fr
} the original work is properly cited. 


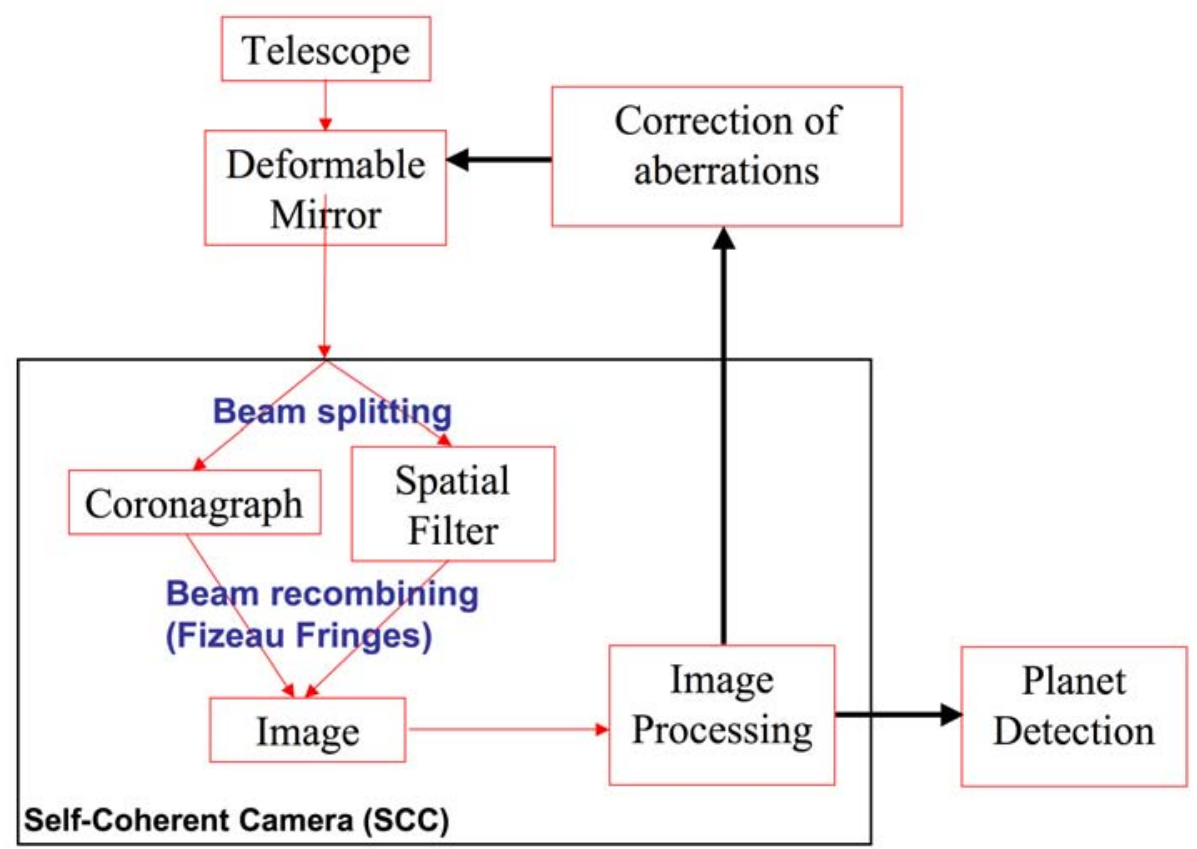

Fig. 1. Principle of the Self-Coherent Camera. See text for more details.

\section{Self Coherent Camera Principle}

The goal of the self-coherent camera is the measurement of phase and amplitude aberrations in the pupil upstream the coronagraph and the speckle field estimation directly from the coronagraphic science image. To do it, we use spatial interferences in the science image to encode the stellar speckles which are directly linked to the wavefront aberrations. This section briefly recalls the principle of the self-coherent camera (SCC) which can : 1) measure the uncorrected residual phase directly from the final science image 2) extract the image of a planet from the residual speckles. More details can be found in $[13-16,19]$. The figure 1 presents a principle scheme. A deformable mirror, located in a plane conjugated to the entrance pupil, reflects the beam incoming from the telescope. We then split the beam. In one channel, the beam goes through a coronagraph. In the other channel, we suppress all the companion light with a spatial filter and extract a beam containing only light from the hosting star. Finally, we recombine the two beams in a Fizeau scheme to obtain spatial fringes in the science image on the detector. Phase and amplitude aberrations give residual speckles in the last focal plane. Since no companion light is transmitted through the second channel, spatial interferences will only affect stellar speckles (cf. Fig. 2) whereas it does not have any impact on a possible companion image since companion light is not coherent with star light. The stellar speckles are thus spatially encoded (modulated) whereas the companion image is not.

The process for detecting faint companions follows two steps : - First, estimation of phase and amplitude errors from the focal plane image and correction for them using a deformable mirror Second, the aberrations potentially not corrected still appears as fringed speckles on the focal plane and a post-processing algorithm is used to perform the ultimate planet detection. This second step can be compared to the differential techniques described in Sect. 1 using incoherence between the star and the planet to discriminate stellar speckles from a planet. In this paper, we only study the first step of the SCC. The estimation of the phase and amplitude for a fixed wavefront error using the SCC has already been studied in the context of space-based observation $[15,16]$. Compared to classical adaptive optics which are limited by non-common path errors between the wavefront sensor and the science beams, 

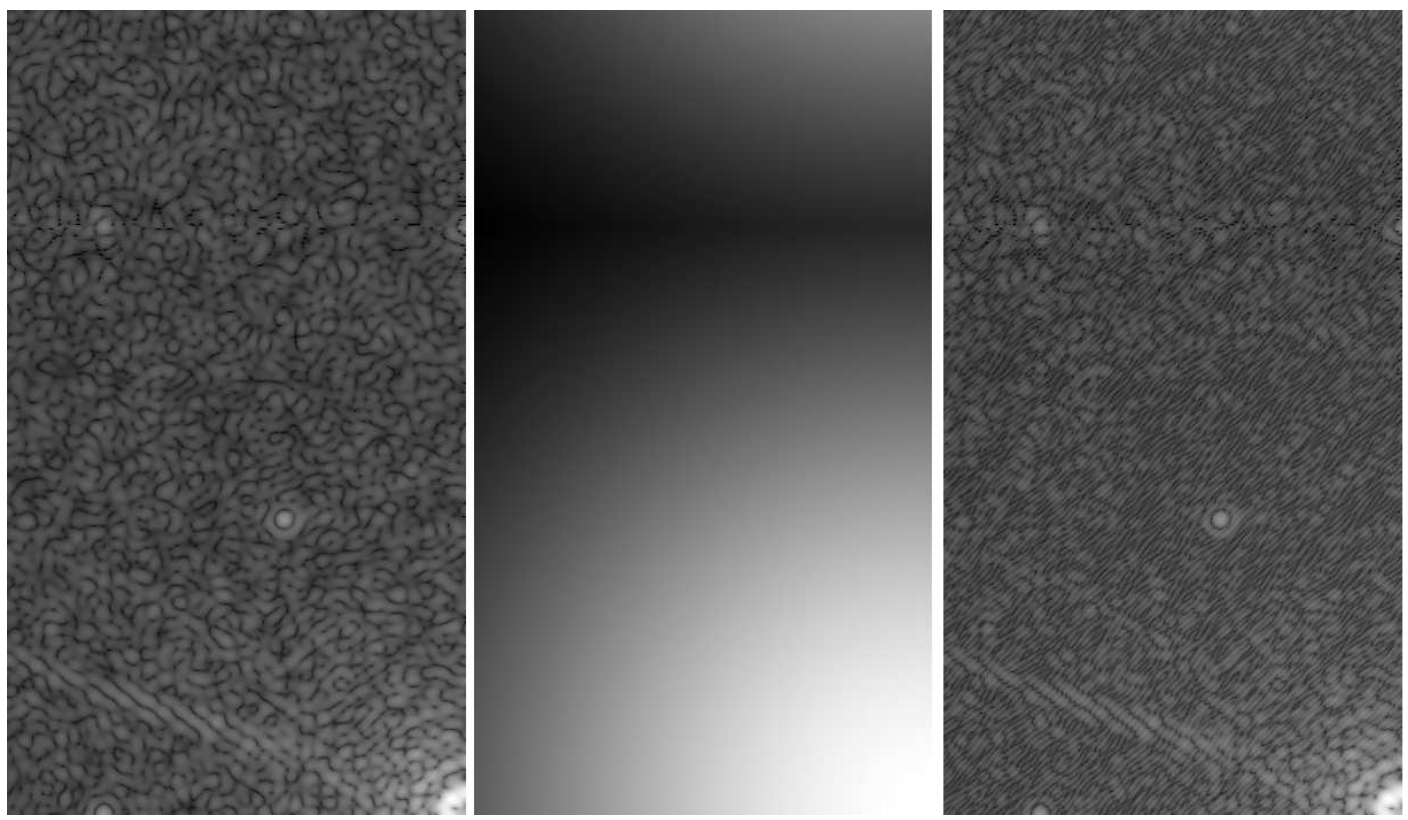

Fig. 2. This figure shows 3 times the same part of the simulated detector for different cases: (a) Image formed after the sole coronagraph for the pupil of diameter D showing the residual speckles. (b) Image of the sole spatially filtered channel for the pupil of diameter $D_{R}$. (c) Interferential image (science image) where the speckles are spatially encoded by fringes. The spatial scale is the same for (a) and (c) and has been enhanced for (b) to better see the intensity distribution of this channel. Only part of the detector is shown. Center of all images (corresponding to coronagraph axis) is located on the lower right side. Diffraction effects from spider arms and holes between segments are visible in images (a) and (c).

the SCC avoid that problem by estimating wave-front errors from the encoded residual speckles of the interferential image (Fig. 2, c) which is also the science image.

The mean intensity of residual speckles of the coronagraphic channel is relatively flat over a field of view of diameter of a few tens of $\lambda / \mathrm{D}$ and it is also very attenuated because of the coronagraph. To optimize fringe contrast, we match intensity distributions and fluxes of both channels. To do so, we decrease the diameter of the diaphragm of the spatially filtered channel $\left(D_{R}\right)$ compared to diameter of the coronagraphic pupil (D) in order to obtain an almost flat intensity in the focal plane $\left(D>>D_{R}\right)$. We can notice that this diaphragm reduces the impact of aberrations on this reference channel since only a few $\lambda / D_{R}$ are visible in the image. Thus, this channel is not very sensitive to aberrations and can be calibrated before the interference recording [16]. Fluxes are equalized using a variable neutral density or by condensing the light in the Reference channel before the pinhole. Several technical setup has been proposed based on classical interferometry or exploiting diffraction characteristic of focal mask coronagraphs $[15,16]$. In Figure 3 , we present, at the same spatial scale and for an instantaneous snapshot after an extreme adaptive optic system used on the E-ELT, an image after the coronagraph which shows residual speckles, an image corresponding to the reference channel for a pupil of diameter $D_{R}=D / 85$ and an interferential image where the residual speckles are spatially encoded by fringes.

\section{Sensing the static aberration}

While the SCC appears as a very promising approach for high precision adaptive optics (AO) on spacebased telescope, it can certainly not replace a classical AO system on ground-based telescope. Indeed, the computation time needed and more importantly the chromatic limitation of the instrument which requires the use of a Wynne corrector [18] or the use of an integral field spectrometer implies that it is less sensitive than classical AO for fast phase measurements. Besides, SCC requires about three 


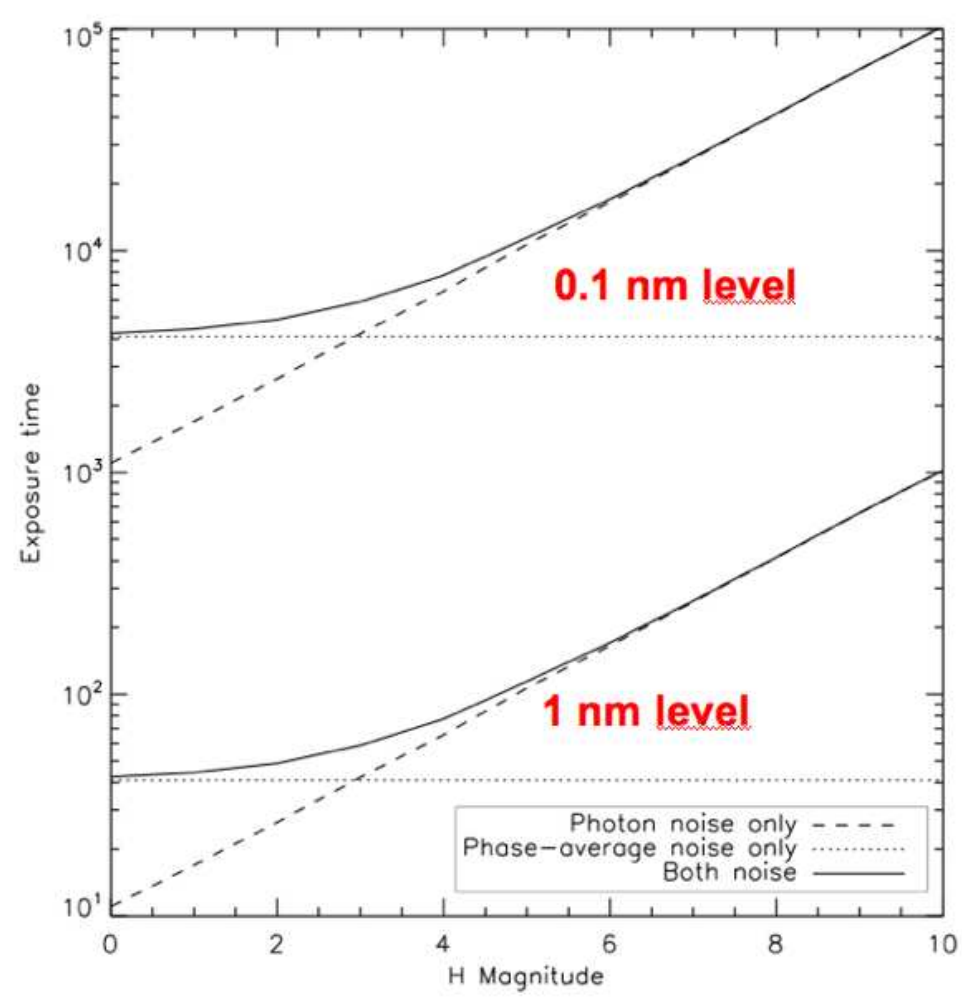

Fig. 3. Exposure necessary to measure static aberration with levels of $1 \mathrm{~nm}$ RMS and $0.1 \mathrm{~nm}$ RMS as a function of magnitude. Curves show photon noise limitation and phase-averaging limitation. The spectral bandwidth simulated is a narrow band at the beginning of the $\mathrm{H}$ band: $\lambda_{0}=1.6 \mu \mathrm{m}, \frac{\lambda}{\Delta \lambda}=100$. The level of residual aberrations is measured in the central $90 \%$ of the corrected area.

times more pixels than other wavefront sensor, implying a larger sensitivity to detector read out noise. However, since it is able to measure the aberrations in coronagraphic images and directly from the final images, it appears as a very interesting instrument to estimate precisely the static aberrations, which are strong limitations in high dynamical range imaging. To evaluate the capability of the SCC as a slow wavefront sensor coupled with a fast extreme AO, we numerically simulated an XAO system applied on the E-ELT and introduced small static aberrations. The simulation assumes an E-ELT equipped with a deformable mirror made of 210x210 actuators and a Self-Coherent Camera. The Self Coherent Camera is simulated assuming a perfect coronagraph. The spatially filtered channel is assumed to be perfect with a diaphragm size $D_{R}=D / 85$. No detector noise is taken into account. The turbulent residual after XAO correction of $64 \mathrm{~nm}$ RMS is derived from the Power Spectral Density (PSD) of the extreme adaptive optics calculated by the EPICS team [5]. For this first simulation, the evolution of turbulence is simulated by using independent wavefront maps that are supposed to last the lifetime of the coherent time (here $10 \mathrm{~ms}$ at the working wavelength bandwidth $\mathrm{H}: \lambda_{0}=1.65 \mu \mathrm{m}, \Delta \lambda=0.35 \mu \mathrm{m}$ ). The static aberration PSD evolves as $\mathrm{f}^{-2}$ where $\mathrm{f}$ is the spatial frequency.

First, the amplitude of static aberrations is set to $10 \mathrm{~nm}$ RMS and we estimate the time necessary to decrease this aberration to 1 and $0.1 \mathrm{~nm}$ RMS for a given star magnitude. The result of this simulation is reported in Figure 2. For very bright stars $(\mathrm{H}<2)$, this time is only limited by the averaging of residual turbulent aberrations. As expected, we found that this residual noise on the phase estimation is linearly proportional to the amplitude of turbulent residual aberrations after XAO and inversely proportional to the square root of the number of independent turbulent simulated phase screens. For fainter stars, photon noise dominates the noise from the averaging of residual turbulent aberrations. The results are very promising since it appears that we can reach a low level of optical defects $(1 \mathrm{~nm}$ 


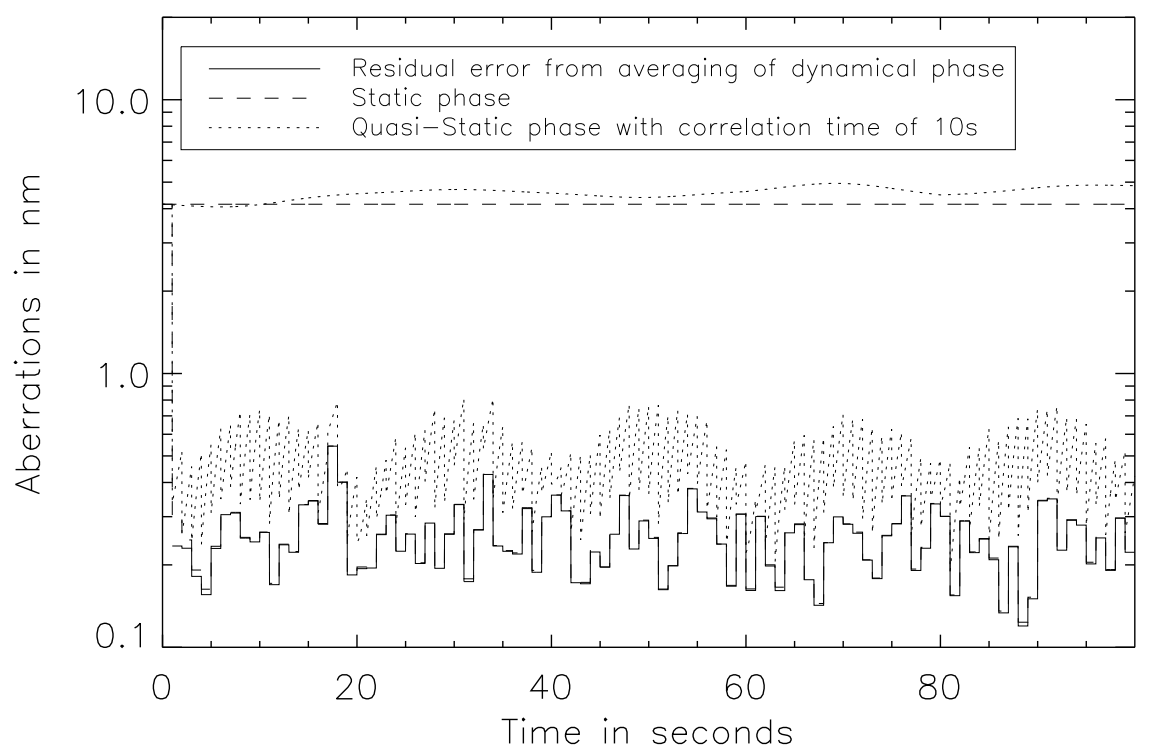

Fig. 4. Amplitude defects (top) and estimation errors (bottom) for static aberrations (dashed line) or quasi-static aberrations (doted line). The amplitude of the defects and the estimation errors are both given in nm RMS. The residual error from averaging of the dynamical turbulent phase is also shown (solid line).

RMS) in a relatively short time (a few hundreds of seconds) for the expected average magnitude target. As reported in Cavarroc et al. [11], the theoretical level of residual speckle varies as the inverse of the square of the RMS amplitude of these aberrations. So decreasing this level to $1 \mathrm{~nm}$ RMS brings a gain of almost 500 compared to the hypothesis of $20 \mathrm{~nm}$ RMS [11] and the detection of rocky-planets seems now possible.

\section{And if static is not static ?}

Above, we took the hypothesis that the optical aberrations created in the telescope was fully static. Unfortunately, this is optimistic and we need to study the impact of long or short term variation of these quasi-static aberrations. To do so, a second simulation has been developed. We also changed the way the turbulent phase aberrations is simulated. Instead of simulating independent phase screens every $10 \mathrm{~ms}$, we simulate complete turbulent layers and translate them in front of our numerical pupil. For calculation time reason, we did not simulated an E-ELT XAO but instead used the SPHERE/XAO configuration (40x40 actuators over a $8 \mathrm{~m}$ diameter pupil). The sampling in the pupil plane is $10 \mathrm{~cm} / \mathrm{pixel}$. We simulate 2 orthogonal layers with a Fried parameter $r_{0}=1 \mathrm{~m}$ in the observing wavelength (H band), an outer scale of $20 \mathrm{~m}$, and a wind speed of $10 \mathrm{~m} / \mathrm{s}$. To simulate the AO correction, first, we estimate the phase measured by a wavefont sensor limited to spatial frequencies that the deformable mirror can correct $(40 \mathrm{x} 40)$. This estimation is calculated by a Fourier filtering of the needed spatial frequencies. Then, we apply this correction of the phase but with a temporal delay of $1 \mathrm{~ms}$ (it is our sole error in this case). The residual aberration amplitude over the pupil after correction is then approximately of $35 \mathrm{~nm}$ RMS. We simulate 100 images per second and integrate these $100 \mathrm{SCC}$ images before calculating the phase estimation by the SCC algorithm. From this $1 \mathrm{~s}$ image, we estimate the measurement errors for static aberrations and for quasi-static aberrations. The quasi-static aberrations are simulated as a combination of static aberrations so that the phase screen is completely uncorrelated after 20 s. The complete simulation data reproduces a 100s observation. No photon noise is introduced in this simulation. 
While the estimation errors for static aberrations are limited by the turbulent phase measurement (figure 4), the quasi-static variation introduces an error for the estimation of the phase. Its level is however relatively low ( $0.45 \mathrm{~nm}$ RMS compared to $0.25 \mathrm{~nm}$ RMS for fully static aberrations) for an amplitude of the quasi-static aberrations of $5 \mathrm{~nm}$. If the phase errors that need to be corrected have a longer correlation time, it is possible to increase the exposure time used to estimate the aberrations and then improve the SCC phase estimation. The correlation time and amplitude of the quasi-static aberrations are poorly constrained. We expect to get better information on this with SPHERE for the VLT. A good estimation of these values in the case of the E-ELT is also necessary to calculate more precise capability of high contrast imaging system like EPICS.

\section{Conclusion}

The concept of the Self-Coherent Camera (SCC) is very new and a lot of area are still to be studied in details. While it has been proposed for space based observation, where it appears to be very promising, use on ground based instrument seems also feasible. It is especially important in the context of EPICS, the planet finder of the E-ELT. Indeed, this ambitious project definitely needs an efficient solution to estimate the static aberrations. The solution proposed by the SCC to measure the phase directly from science image is particularly appealing. In this paper, preliminary simulations has been described. It appears that the SCC is able to measure static or quasi-static aberrations efficiently. The level reachable by the SCC depends on several criteria, two of them being poorly constrained : the amplitude and correlation time of quasi-static aberrations. Measurement of these values are needed to estimate more precisely the efficiency of high contrast imaging instrument like the SCC on the E-ELT. Also, a more detailed study needs to be carried out to estimate the exact capability of the SCC not only on the static aberration measurement but also on the planet detection, which has not been discussed in this paper. Another important step is to set up a laboratory demonstration for the SCC. This has already started and is described in more details in one of the paper of this volume [19].

acknowledgements : The authors are thankful to EPICS team for valuable discussion on SCC concept. Part of this work is supported by European Fund Program 7 : E-ELT Prep Phase grant 211257.

\section{References}

1. Baudoz, P., Rabbia, Y., Gay J., 2000, A\&A141, 319-329

2. Rouan, D., Riaud, P., Boccaletti, A. and Clénet, Y. and Labeyrie, A., 2000, PASP, 112, 1479-1486

3. Boccaletti, A., Riaud, P., Baudoz, P., et al., 2004, PASP, 116,1061-1071

4. Mawet, D. and Riaud, P. and Absil, O. and Surdej, J., 2005, ApJ, 633, 1191-1200

5. Vérinaud, C., Korkiakoski, V., Yaitskova, N., Martinez, P. et al., 2008, SPIE conference, 7014, $70141 \mathrm{~J}$

6. Macintosh, B., Poyneer, L., Sivaramakrishnan, A., Marois, C., 2005, SPIE conference, 5903, 170177

7. Marois, C., Doyon, R., Racine, R., and Nadeau, D., 2000,PASP, 112, 91-96

8. Baba, N., Murakami, N., 2003, PASP, 115, 1363-1366

9. Marois, C., Lafrenière, D., Doyon, R., Macintosh, B., Nadeau, D., 2006, ApJ, 641, 556-564

10. Guyon, O. 2004, ApJ, 615, 562

11. Cavarroc, C., Boccaletti, A., Baudoz, P., Fusco, T., Rouan, D., 2006, A\&A, 447, 397-403

12. Codona, J., Angel, R. 2004, ApJ, 604, L117

13. Baudoz, P., Boccaletti, A., Baudrand, J., Rouan, D., 2006, Proc. IAU Colloquium 200, 553-558

14. Galicher, R., Baudoz, P., 2007, C. R. Physique 8, 333-339

15. Galicher, R., Baudoz, P., Rousset, G., 2008, A\&A, 448, L9-L12

16. Galicher, R., Baudoz, P., Rousset, G., Totems, J., Mas, M. 2009, accepted in A\&A

17. Kasper, M, Beuzit, J.-L., Verinaud, C., Yaitskova, N. et al., 2008, SPIE conference, 7015, 70151S

18. Wynne, C., 1979, Opt. Comm., 28, 21-25

19. Mas, M., Baudoz, P., Galicher, R., Rousset, G., Baudrand, J., Coton, C., Assémat, F., this volume 